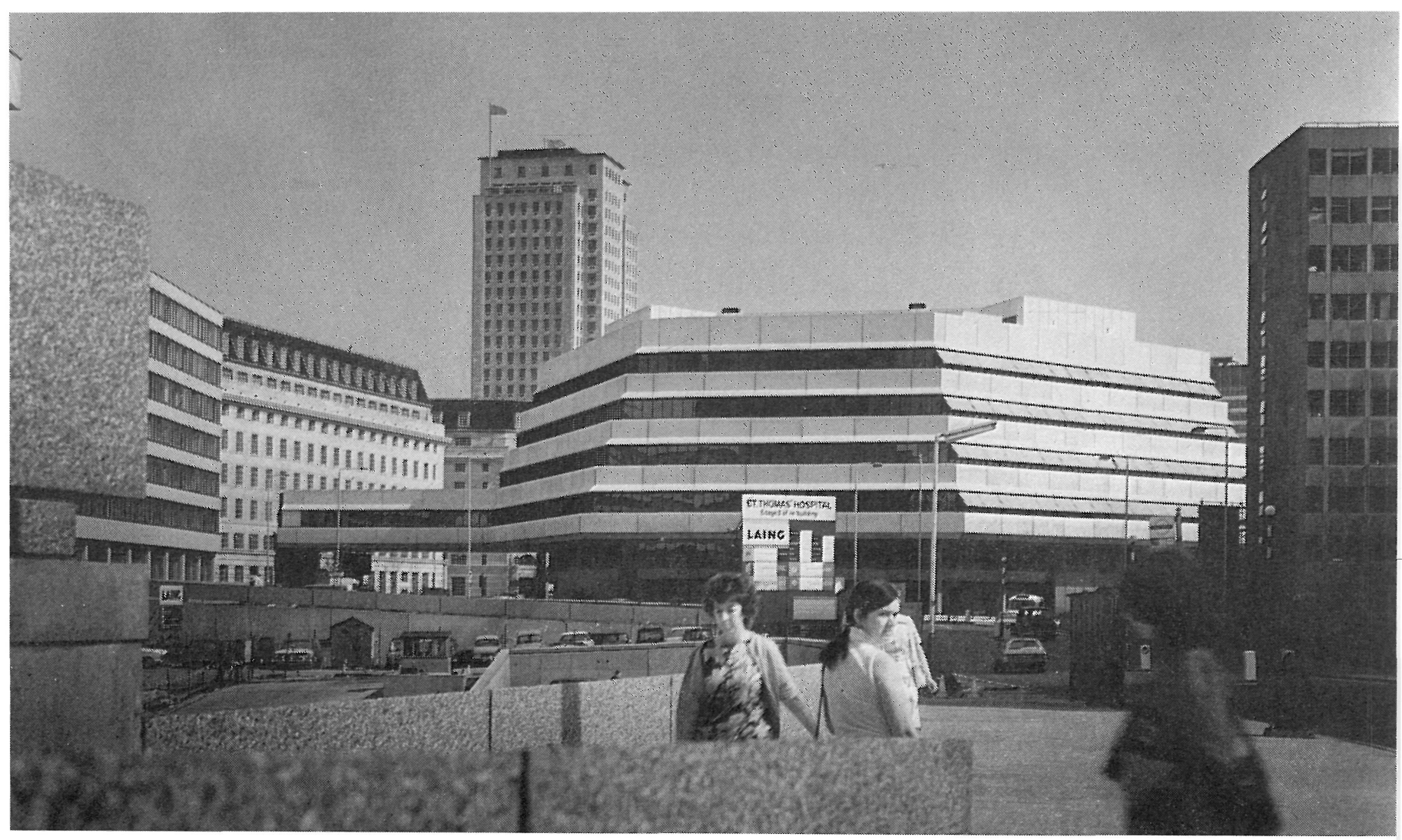

\section{ofieinas Isla}

Londres * Gran Bretaña

SIR ROGER WALTERS,

J. E. KNIGHT y W. J. SUTHERLAND, arquitectos

$131-111$

\section{Sinopsis}

Este edificio de oficinas se levanta sobre un solar de más de $4.000 \mathrm{~m}^{2}$, junto al puenque ocupa el Parlamento y muy próximo Ayuntamiento. Es una construcción de forma hexagonal irregular, que ocupa totalmente el solar. Consta de una planta baja principal y cinco de oficinas, estando las cuatro últimas retranqueadas progresivamente. Cada $y$ pla posee un núcleo central de servicios $y$ comuscaciones, y dos áreas de oficinas hay una terraza ajardinada desde la cual se disfruta de magníficas vistas. A unos $4 \mathrm{~m}$ por debajo del nivel de la calle se halla el sotano con los archivos y un paso de peatones. Desde el patio central del Ayuntamiento se puede llegar, por su puente elevado, al nuevo edificio, que tiene 1.500 puesto






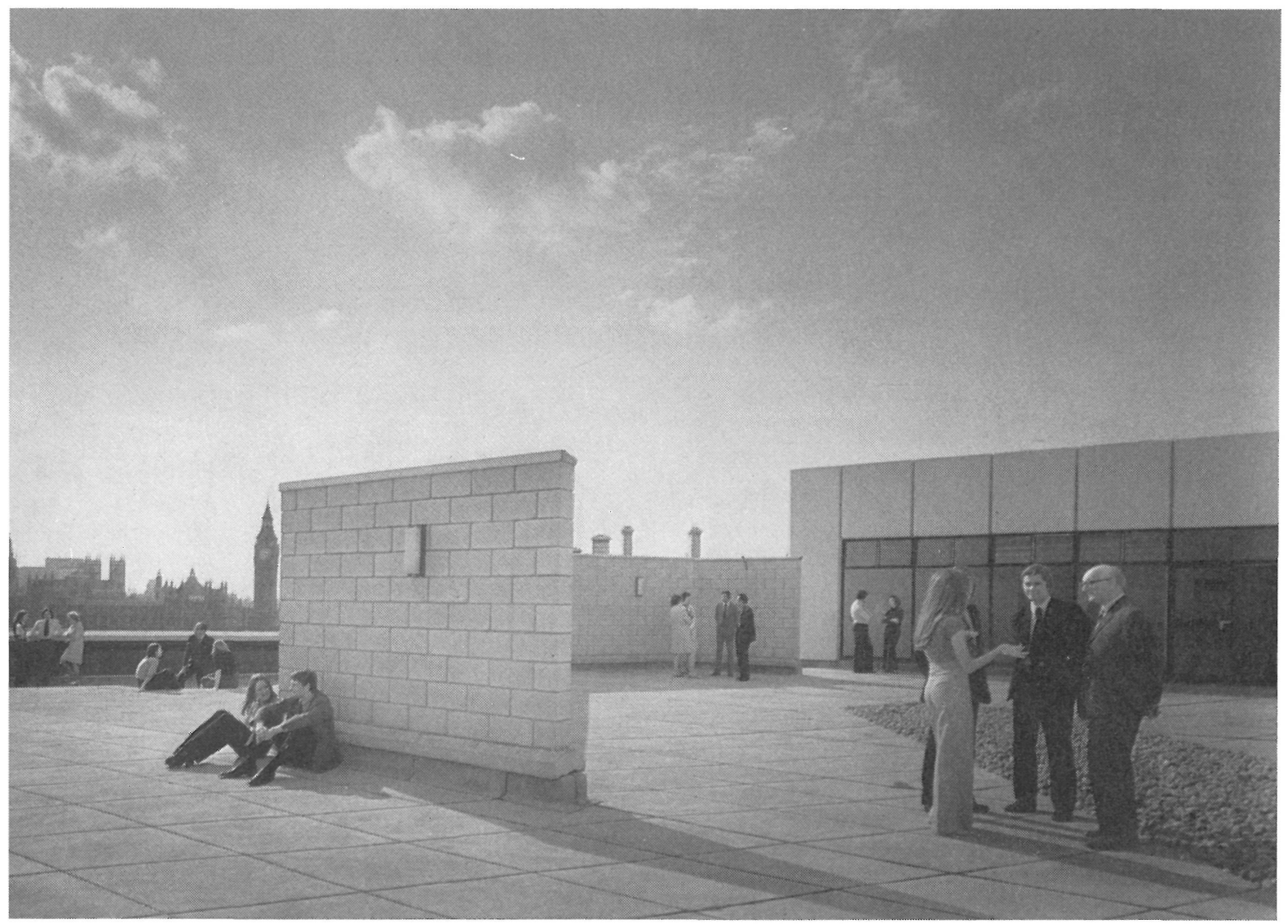

La estructura del edificio es de hormigón armado, con pilares situados de forma que permitan la construcción futura de una vía de circulación subterranea. La del puente consiste en cuatro grandes vigas que apoyan, por un
con rodillos.

Exteriormente la construcción va revestida con piezas prefabricadas de basalto siliceo, dispuestas en bandas continuas perimetrales alternando con las hileras de venta

En el edificio se han utilizado los más modernos sistemas de acondicionamiento de aire, iluminación y seguridad. Asimismo se diseñó esespacios.

El solar elegido para construir las oficinas centrales del Consejo del Area Metropolitana del gran Londres, tiene una superficie de más de $4.000 \mathrm{~m}^{2}$, y está situado en un lugar privilegiado, junto al puente de Westminster, en la orilla opuesta a la que ocupa el Parlamento, y en proximidad al Ayuntamiento de la ciudad.

La superficie disponible y las ordenanzas de altura impusieron grandes limitaciones en el diseño, aumentadas por la necesidad de adecuar la escala del edificio a la de los ya existentes. Por otro lado, la estructura necesitó un planteamiento especial ante la posibilidad futura de construir un paso subterráneo para tráfico a través del solar, y un puente de conexión con otro edificio, para ampliación de las oficinas.

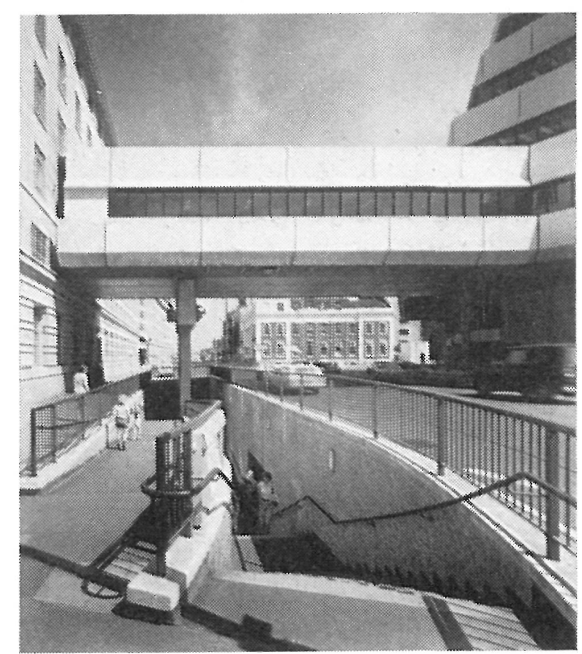


planta a nivel de la calle

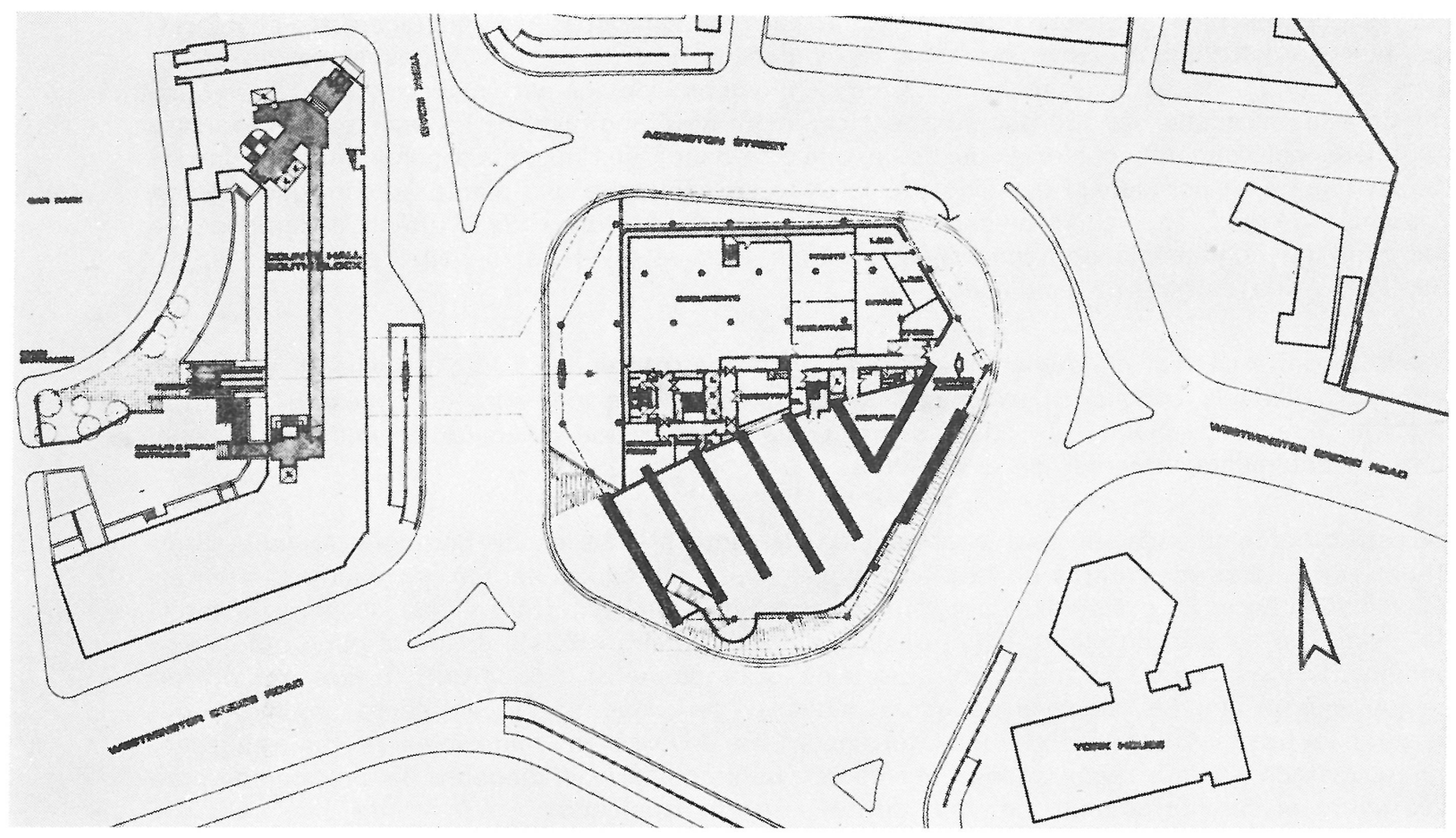

El proyecto consiste en un edificio con planta en forma de hexágono irregular, que aprovecha el solar al máximo, al tiempo que aminora el impacto visual que produce su volumen. Posee una planta principal y cinco de oficinas, de las cuales las cuatro últimas están retranqueadas progresivamente, dejando unos pasillos perimetrales que facilitan la limpieza y mantenimiento de las ventanas. Cada planta consta de un núcleo compuesto por los siguientes elementos:

planta de sótano

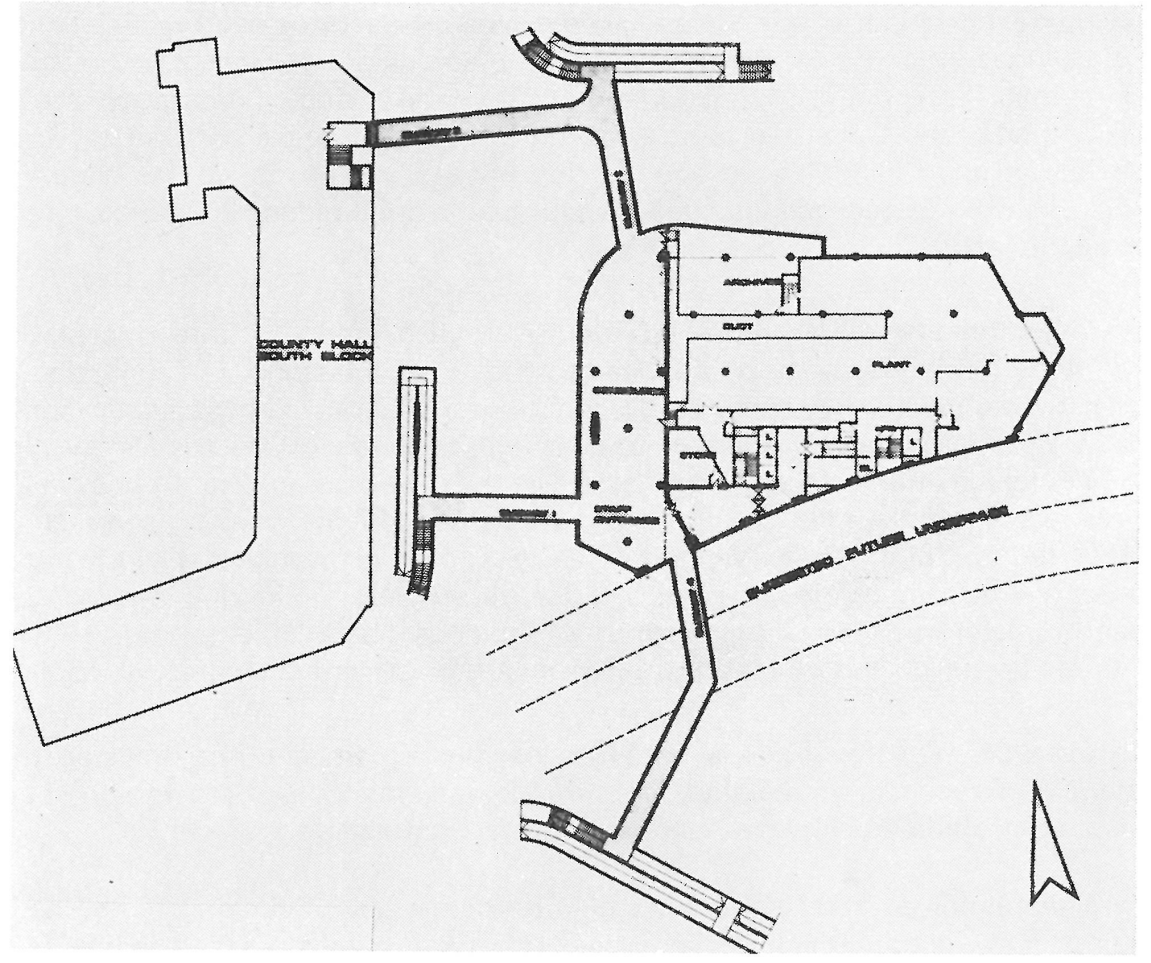


tres ascensores para personas y uno pequeño para documentos, un montacargas, escaleras, vestíbulo de distribución, servicios, salas de conferencias y de lectura, y despachos de ejecutivos. Las áreas de oficinas se hallan situadas a ambos lados de este núcleo, conteniendo cada una de ellas alrededor de 120 puestos de trabajo. En el último nivel se ha realizado una terraza ajardinada parcialmente cubierta, desde la que se domina una magnífica panorámica de la ciudad. A unos $4 \mathrm{~m}$ por debajo del nivel de la calle se encuentra una planta sótano, que alberga el archivo general, un archivo mecanizado y un paso de peatones. Este último desemboca en una zona de confluencia para personas, también bajo el nivel de la calle, en la que se ha emplazado una entrada de empleados.

Desde el patio central del Ayuntamiento se puede llegar al nuevo edificio mediante una escalera mecánica que conduce a un puente sobre la calle. Este elemento de conexión se utiliza también para la instalación de oficinas complementarias, y fue diseñado de manera que quedara perfectamente integrado en el conjunto.

La estructura empleada en esta construcción de siete plantas es de hormigón armado, constituida por pilares dispuestos en la planta hexagonal, a distancias de $9 \mathrm{~m}$, y en centros de triángulos equiláteros. Este sistema permitirá construir el paso subterráneo, que ocupará un tercio del solar con su trazado curvo, hacia el que podrá dirigirse el tráfico desde el puente de Westminster. Los pilares de la zona central son de gran diámetro y longitud; fueron estudiados, conjuntamente con los técnicos de obras públicas, para que aparezcan como columnas que surgirán a través del futuro paso subterráneo. Los ensayos de materiales fueron realizados por el método sónico, registrándose los datos obtenidos para así disponer de información práctica sobre el comportamiento del tipo de cimentación empleado.

Antes de proceder a la excavación del terreno se construyó un muro de contención de la calzada, en el perímetro que fue necesario. También se colocaron puntales y otras protecciones para impedir el deslizamiento de las construcciones vecinas y de los pasos de peatones existentes. El subsuelo, por su proximidad al río, estaba totalmente inundado, lo que obligó a instalar un completo sistema de bombeo durante los trabajos, para achicar el agua.

La estructura del puente sobre la calle se compone de cuatro vigas prefabricadas, apoyadas en el nuevo edificio, por un lado, y en soportes sobre rodillos, por el otro, lo que permite dejar un tramo en voladizo que se adosa, sin apoyar, en la estructura del edificio del Ayuntamiento. Estas vigas principales soportan otras transversales, formando un entramado para el reparto de cargas. Este diseño tan simple fue adoptado, principalmente, para evitar al máximo las interrupciones del tráfico en la calle durante la construcción del puente. Con este mismo motivo se fijaron unas bandejas de acero a los bordes inferiores de las vigas principales, formando una especie de marquesina que aseguraba la inmunidad del tráfico mientras los trabajos continuaban arriba.

El revestimiento exterior del edificio se realizó mediante piezas prefabricadas, de 4,60 $\mathrm{m}$ de longitud, fabricadas a base de basalto silíceo machacado y calcinado, que les confiere una gran resistencia a los cambios de temperatura. Están dispuestas en bandas continuas perimetrales, proporcionando, además, recubrimiento a los conductos de aire acondicionado y a los pequeños voladizos que ayudan al retranqueo de las cuatro últimas plantas. Se alternan en cada nivel con bandas de ventanas de vidrios dobles y marcos de aluminio, que reducen el nivel del ruido exterior. Mediante la coloración al bronce del vidrio, se atenúa el deslumbramiento y el calentamiento en el interior del edificio, a lo que ayudan, también, las persianas semitransparentes de color naranja, articuladas hacia el exterior, y accionadas artificialmente por medio de cuatro células fotoeléctricas que se activan con la luz solar, situadas en la terraza.

La falta de espacio obligó a estudiar las cinco plantas de oficinas de forma que la superficie fuera aprovechada al máximo, procurando amplias zonas de trabajo, pero con una ambientación controlada que creara una atmósfera confortable.

Para disminuir el nivel sonoro en el interior de las oficinas, los techos suspendidos de cada planta son acondicionadores acústicos $\mathrm{y}$, además, todas las principales zonas se encuentran 


\section{planta primera}

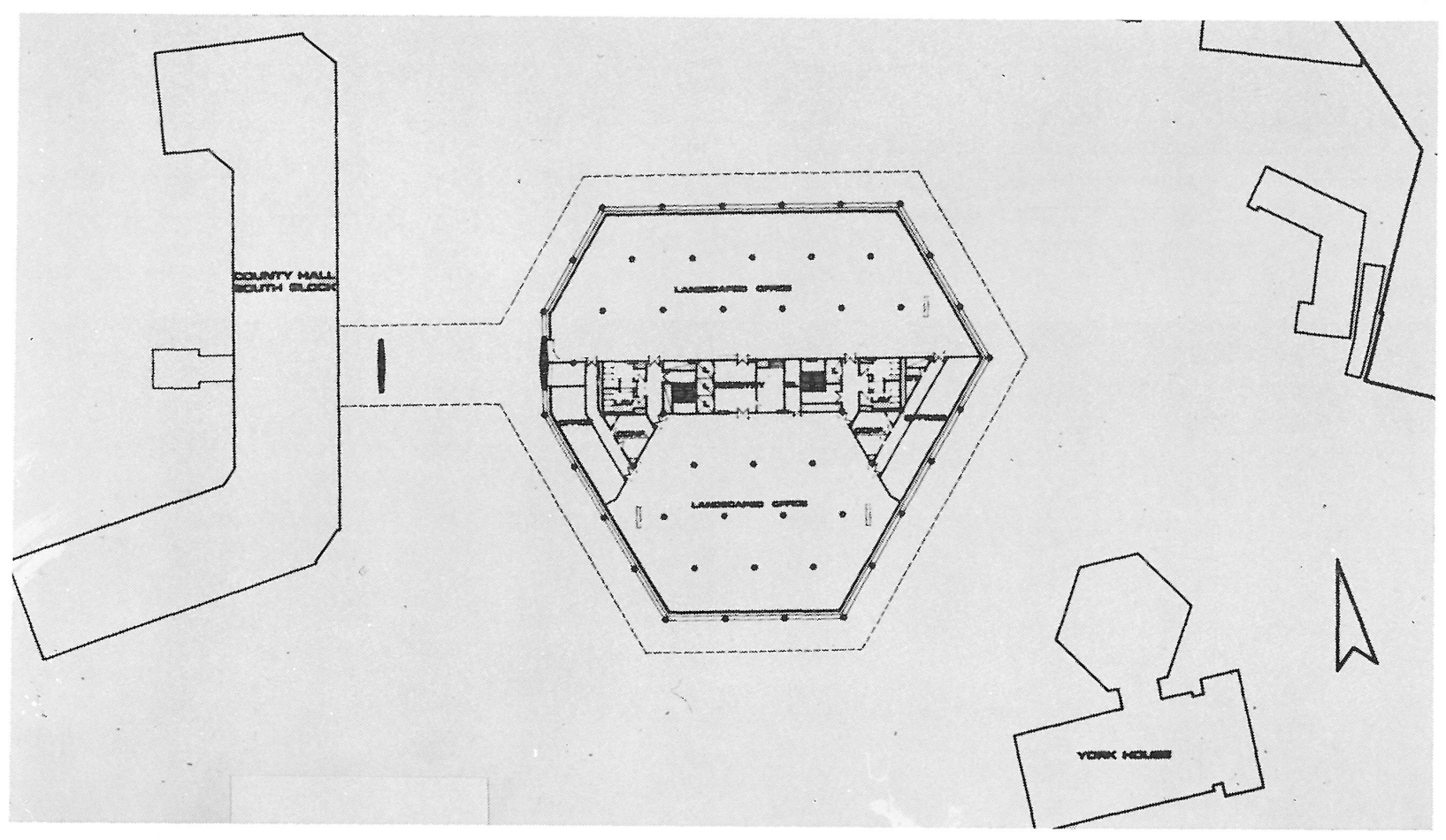

enmoquetadas. El tratamiento interior se ha realizado a base de particiones de yute lavable, superficies de materiales plásticos y entarimados que, junto con las columnas de hormigón visto, ayudan a un fácil mantenimiento. El mobiliario se diseñó especialmente para que permitiera localizar las diversas zonas y facilitar la relación entre las distintas instalaciones. Pan-

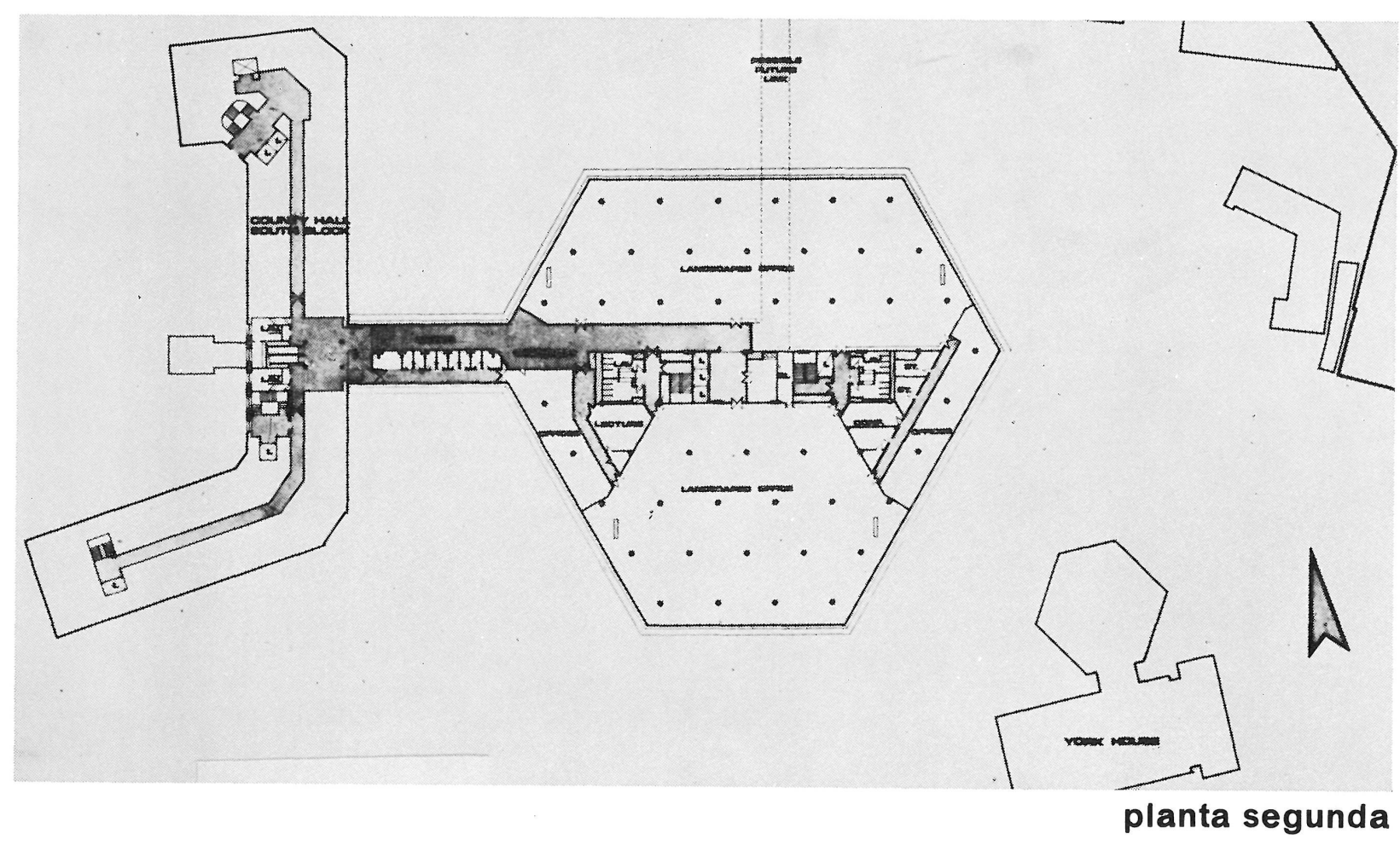



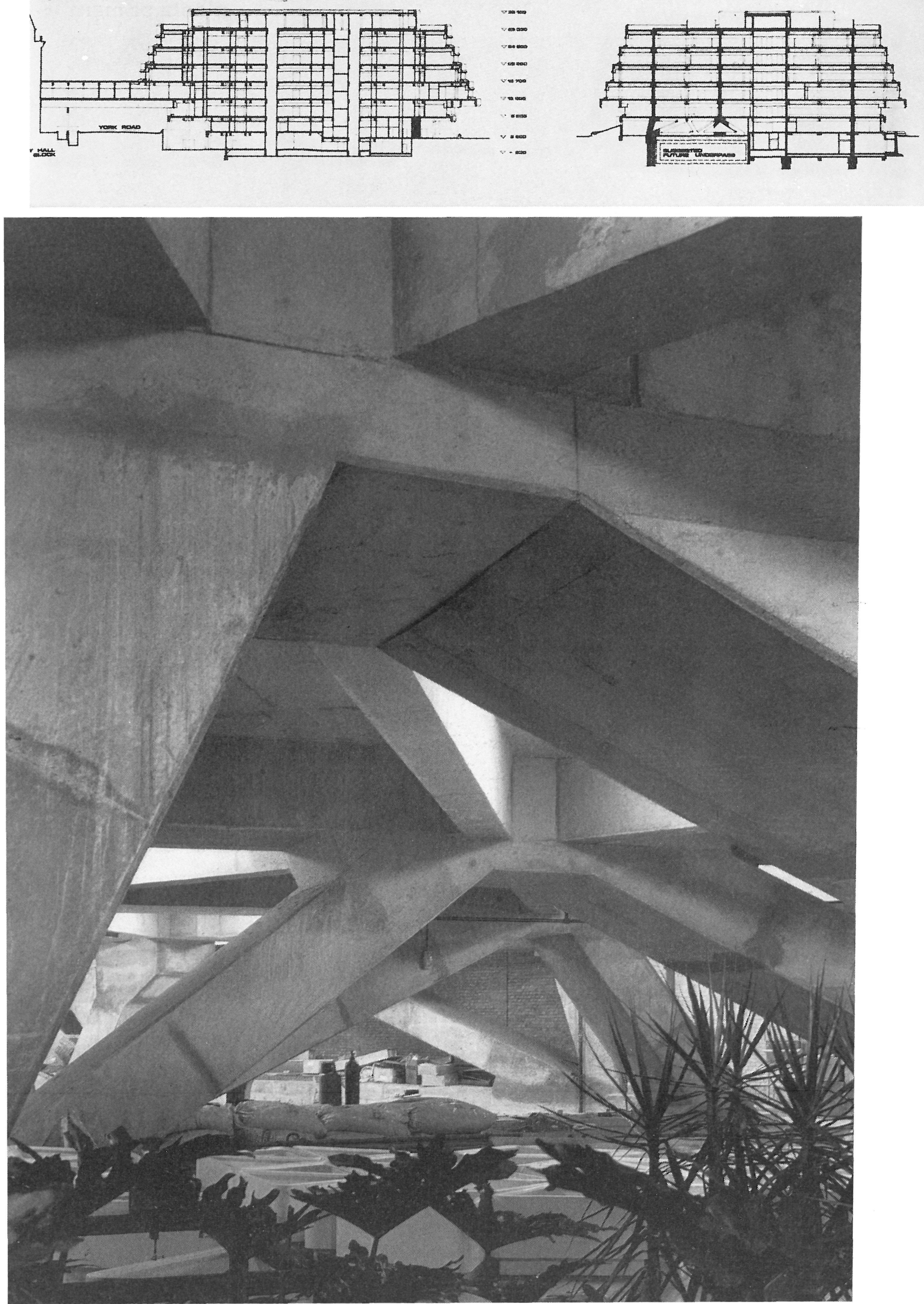




tallas de colores y plantas de interior, proporcionan interés visual y completan el esquema de las vías interiores de circulación. Se han dispuesto también áreas de descanso, con máquinas automáticas y armarios individuales. Entre los lavabos se dejó un espacio cerrado visitable que permite dejar las conducciones vistas para su inspección, así como instalar el jabón líquido y las papeleras.

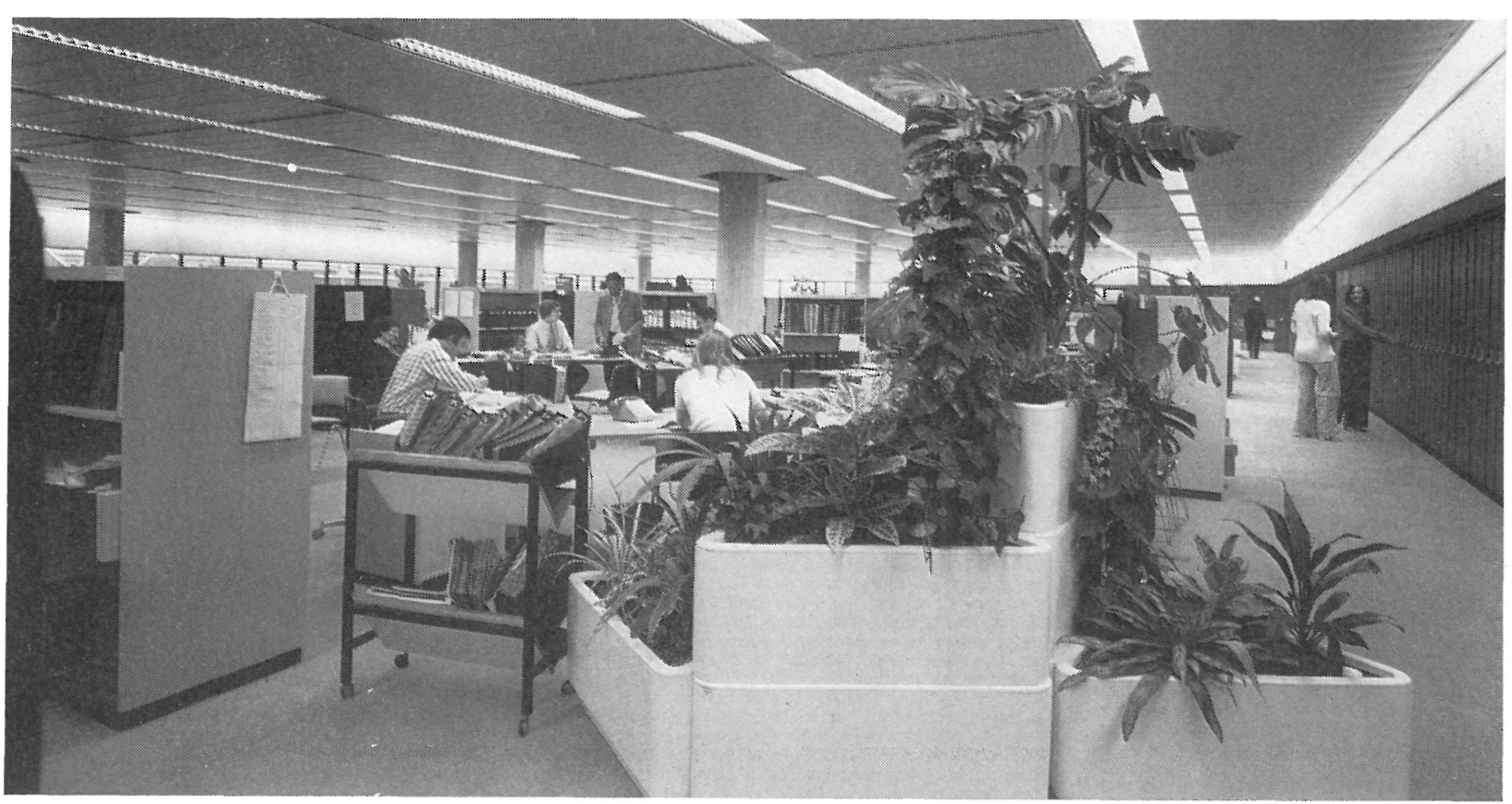

FOTOS : RICHARD EINZIG BRECHT-EINZIG LIMITED

El aire, la temperatura y la humedad correctos se obtienen según el principio de recuperación del calor. El aire es filtrado, enfriado o calentado, hidratado o secado, según las necesidades de cada momento, por un equipo de tratamiento. Después es llevado por conductos verticales y horizontales, que fueron previstos al hacer el diseño de la estructura. Se descarga en las diferentes áreas del edificio mediante unidades montadas bajo las ventanas o desde difusores dispuestos en el falso techo. Posteriormente es extraído por arriba, entre las rejillas de los 
puntos de luz, y llevado de nuevo al equipo de tratamiento, para su recirculación, o a la atmósfera, según se requiera. Tres enfriadores de agua, eléctricos, proveen a las seis unidades de tratamiento que componen el equipo. Los dos calentadores de gas, para la calefacción y el agua caliente, se hallan situados en la zona de confluencia peatonal, bajo el nivel de la calle.

La iluminación, fluorescente y de gran eficacia, se conecta y desconecta de manera automática a horas previamente determinadas. En los suelos de cada planta se instalaron rejillas de suministro con los enchufes de luz y teléfono, a intervalos de $2 \mathrm{~m}$, para dar un máximo de flexibilidad en la colocación de los lugares de trabajo. Los cables de las instalaciones se conducen, mediante tubos protectores, a través de las estructuras.

Un último detalle del edificio, en el que trabajan unas 1.500 personas y que responde a la necesidad de proporcionar un fácil acceso al público, es su iluminación exterior, emplazada bajo la segunda planta, y que le confiere un carácter de ligereza a pesar de su volumen.

Arquitectos colaboradores:

GEOFFREY F. HORSALL, B. ARCH, ERIC HAYES, ALAN STOCKLEY y BHS THAXTON

\section{résumé}

Bureaux "Island" Londres - Grande-
Bretagne
Sir Roger Walters, J. E. Knight et W. J. Sut-
herland, architectes

herland, architectes

Ce bâtiment de bureaux est situé sur un terrain de plus de $4.000 \mathrm{~m}^{2}$, près du pont de Westminster, sur la rive opposée à celle occupée par le Parlement et tout près de sous forme hexagonale irrégulière, que occupe tout le terrain. Elle se compose d'un rez-de-chaussée et de cinq niveaux pour les bureaux, dont les quatre derniers sont progressivement en retrait. Chaque niveau a un noyau central de services et communications, et deux zones de bureaux situées aux deux côtés. Le dernier niveau comporte une terrasse aménagée en jardin, d'où l'on jouit d un beau panorama. A environ $4 \mathrm{~m}$ le sous-sol abritant les archives et un pas. sage pour piétons. Un pont surélevé conduit de la cour centrale de I'Hôtel de Ville au nouveau bâtiment, ayant 1.500 postes de travail.

La structure du bâtiment est en béton armé, avec des poteaux situés de facon à permettre la construction future d'une voie de circulation souterraine. La structure du pont surélevé consiste en quatre grandes poutres qui reposent, d'une part, sur le nouveau bâ. à rouleaux.

A l'extérieur, le bâtiment est revêtu de pieces prefabriquées en basalte siliceux, ques alternant avec les files de fenêtres. Les systèmes les plus modernes de conditionnement d'air, d'éclairage et de sécurité ont été utisés pour ce batiment, dont le mobilier a été particulièrement conçu pour
différencier et rattacher les divers espaces.

\section{summary}

The "Island" Offices - London Great Britain

Sir Roger Walters, J. E. Knight and W. J. Sutherland, architects

This office building occupies an approximately $4,000 \mathrm{~m}^{2}$ site, situated at the Westminster Bridge, opposite the Houses of Parliament and the County Hall. The design
is an irregular hexagon which takes full is an irregular hexagon which takes full
advantage of the site area. II comprises advantage of the site area. II comprises
one basement, one main floor and five office floors, the latter ones being terraced. On each storey a services and communications nucleus has been installed and on either side of this nucleus the offices are located. On the top floor there is a roof garden with a magnificent view. About $4 \mathrm{~m}$ below stree level is the basement with the archives and the pedestrian subway. From the central courtyard of the Country Hall direct access has been provided to the new building vi staff of about 1,500 people.

The structure is of reinforced concrete with columns situated in such a way that provision has been made for constructing future underpass. The bridge consists of four beams which at one end rest upon bearings.

The outside surface is covered with prefabricated siliceous basalt panels, placed in the aluminium framed windows.

The building is provided with the most modern systems for air-conditioning, lighting and safety. Moreover, the furniture has barious spaces from ned to dher to them to each other.

\section{zusammenfassung}

Die «Insel» Büros - London - Grossbritannien

Sir Roger Walters, J. E. Knight und

W. J. Sutherland, Architekten

Dieses Bürogebäude is auf einem etwa $4.000 \mathrm{~m}^{2}$ grossen Grundstück, neben der Westminster Brücke, gegenüber von dem Rathaus, errichtet worden. Es ist eine unregelmässige sechseckige Konstruktion, die das ganze Grundstück einnimmt. Es umfasst
Kellergeschoss, Erdgeschoss und fünf BuroKellergeschoss, Erdgeschoss und fün BuroJede Etage ist mit einem Mittkern fü Service- und Kommunikationseinrichtungen versehen, an dessen beiden Seiten die Bürolokale gelegen sind. Im Obergeschoss befiendet sich ein Dachgarten mit schönem Blick auf die Stadt. $4 \mathrm{~m}$ unter der Erde im Kellergeschoss sind die Archiven und die Fussgangerunterführung. Von dem Innen hof steht das mittelst einer Brücke DirektBürindug mit derst neupa

Die Struktur ist aus Stahlbeton mit den Pfeilern so geplant, alass es moglich sei wird, eine zukunftige Verkehrsunterführung Trägern, die an dem einen Ende von dem neuen Bau gestützt werden und an dem an deren, von Rollenlagern.

Die Aussenseite ist von Kieselfertigelemen ten bedeckt, die sich mit den in Aluminium eingerahmten Fenstern abwechseln.

Ferner ist der Bau mit den modernsten Klima-, Beleuchtungs- und Sicherheitsanlagen ausgerustet. Weiterhin sind die Miobel besonheiten entweder zu unterscheiden oder zu verbinden. 